\title{
On Automating Triangle Constructions in Absolute and Hyperbolic Geometry*
}

\author{
Vesna Marinković[0000-0003-0526-899X] \\ Faculty of Mathematics \\ University of Belgrade, Serbia \\ vesnap@matf.bg.ac.rs
}

\author{
Tijana Šukilović[0000-0001-6371-3081] \\ Faculty of Mathematics \\ University of Belgrade, Serbia \\ tijana@matf.bg.ac.rs
}

\author{
Filip Marió [0000-0001-7219-6960] \\ Faculty of Mathematics \\ University of Belgrade, Serbia \\ filip@matf.bg.ac.rs
}

\begin{abstract}
We describe first steps towards a system for automated triangle constructions in absolute and hyperbolic geometry. We discuss key differences between constructions in Euclidean, absolute and hyperbolic geometry, compile a list of primitive constructions and lemmas used for constructions in absolute and hyperbolic geometry, build an automated system for solving construction problems and test it on a corpus of triangle-construction problems. We also provide an online compendium containing construction descriptions and illustrations.
\end{abstract}

\section{Introduction}

Ruler $^{1}$ and compass triangle constructions (abbr. RC-constructions) have been intensively studied in mathematics and mathematical education since ancient Greeks, for now more than two and a half thousand years. Triangles are also a very nice polygon to develop and test artificial intelligence and automated reasoning tools, and systems that automatically solve RC-construction problems are actively being developed (e.g., ArgoTriCS [10]).

Construction problems may require constructing a triangle given some of its significant points. William Wernick [18] has given an exhaustive catalogue of all such problems formulated over the following significant points: three vertices $A, B, C$, circumcenter $O$, three side midpoints $M_{a}, M_{b}, M_{c}$, centroid $G$, three feet of altitudes $H_{a}, H_{b}, H_{c}$, orthocenter $H$, three feet of internal angle bisectors $T_{a}, T_{b}, T_{c}$, and incenter $I$ (see Figure 1 $\left.\right|^{2}$. All problems require constructing a triangle given its three distinct significant points (from those listed). There are 139 such problems that are significantly different. Some problems are redundant (e.g., the triple $A, B$, and $M_{c}$ is redundant since the midpoint $M_{c}$ can be constructed when $A$ and $B$ are given). Some problems are locus dependent (e.g, the triple $A, B$, and $O$ is solvable only if $O$ belongs to the perpendicular bisector of the segment $A B$ ). When redundant and locus dependent problems are excluded, there remain 114 problems, and Wernick solved 65 of these, leaving the others with an unknown status. In the meantime status of all problems from the Wernick's list has been determined, by either solving them, or proving that required triangles are not constructible by using only

\footnotetext{
*This research is supported by the Serbian Ministry of Education, Science and Technological Development through the University of Belgrade, Faculty of Mathematics.

${ }^{1}$ Usually only a straightedge (ruler with no marks) is allowed.

${ }^{2}$ This notation will be used throughout the paper
} 
ruler and compass: there are 74 solvable problems, 39 unsolvable, 3 redundant and 23 locus dependent problems [17].

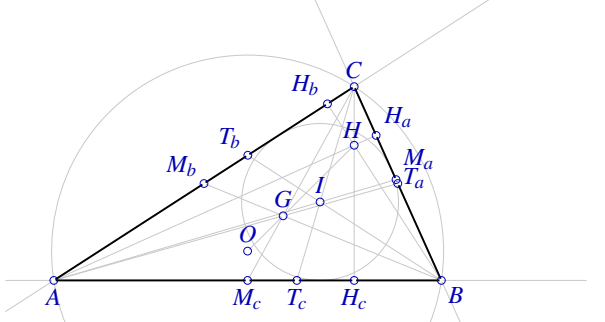

Figure 1: Significant points from Wernick's corpus.

RC-constructions are usually done in the framework of Euclidean geometry. Triangle constructions in other geometries (e.g., hyperbolic) have been studied, but to much lesser extent. In this paper we investigate automated triangle constructions in absolute and hyperbolic geometry (focusing on the Poincaré disc model). We describe an automated solver for those problems, based on our Euclidean triangle construction solver ArgoTriCS. We describe all changes that have been necessary to adapt ArgoTriCS to fully automatically find triangle constructions in absolute and hyperbolic geometry. We apply the modified system to Wernick's corpus and summarize the current results.

\subsection{Related work}

Constructions in hyperbolic geometry. The theory of hyperbolic constructions was almost entirely developed by Russian-writing mathematicians (e.g., Mordoukhay-Boltovskoy [13]). Pambuccian gives constructive axiomatizations of several geometries [14], including Euclidean, absolute and hyperbolic geometry. Unlike traditional geometry axiomatizations (e.g., Hilbert's and Tarski's) that use only relation and no function (operation) symbols, Pambuccian does quite the opposite and gives axiomatic systems that use only function and no relation symbols. There are several toolboxes in dynamic geometry systems (GeoGebra, Geometer's Sketchpad, Cinderella, etc.) that facilitate manual step-by-step constructions in Poincaré disc model.

Automated triangle constructions. Despite long tradition of RC-constructions, there are quite few systems that automate them. Schreck developed Progé [15] - a general framework implemented in PROLOG where different kind of geometric objects (not only triangles) can automatically be constructed. Gao and Chou applied algebraic approach and used Wu's method [19] or Gröbner bases [3] to find locations of unknown objects from the locations of known objects and determine RC-constructibility [5]. Schreck [16] also focused on the algebraic approach and compared Gao and Chou's method with the Lesbegue's method using them to show non-constructibility, but also to extract some RC-constructions from algebraic methods. Gulwani et al. [6] formulated a logic and a programming language of geometry constructions and then applied methods for program synthesis to obtain RC-constructions. Marinković and Janičić [9, 10] focused on triangle construction given its significant points. Their system ArgoTriCS is capable of solving 66 out of 74 solvable problems from Wernick's list. It can also detect all redundant and locus dependent problems from this list.

As far as we know, there has been no previous research on automated triangle constructions or automated theorem proving in non-Euclidean geometries. Algebraic approach that is very successful for Euclidean geometry is hard to apply in the hyperbolic setting since distance constraints are not describable using only polynomials (distances involve logarithms of cross-ratio). 


\section{ArgoTriCS}

ArgoTriCS is a PROLOG system that can solve geometry construction problems automatically, given some background geometrical knowledge [10]. It generates informal description of found construction in natural language (in English) and formal description in GCLC language [8], accompanied by a corresponding illustration. It also generates non-degeneracy conditions which guarantee that the obtained solution exists and has support for proving that generated constructions are correct by using OpenGeoProver [11] and provers available within GCLC tool.

The knowledge base that the system requires was identified through a careful analysis of constructions available in the literature. We came up to a core geometrical knowledge needed for solving triangle construction problems from Wernick's corpus. This knowledge was split into three sets: set of definitions, set of lemmas, and set of primitive constructions. We distinguish between instantiated and general definitions and lemmas. For instance, in a given triangle there is only one orthocenter, one centroid, and one circumcenter. Therefore the lemma of Euclidean geometry stating that these three points are collinear and that the centroid is between circumcenter and orthocenter and is twice as far from the orthocenter than from the circumcenter is an instantiated lemma. On the other hand, the lemma stating that the center of a circle belongs to the bisector of an arbitrary chord of that circle is a general lemma, and therefore it is applicable to any chord of any circle. However, before the search for a construction starts, general definitions are instantiated with all relevant objects and added to the knowledge base as derived definitions, and, similarly, general lemmas are instantiated with objects that satisfy their preconditions and added to the knowledge base as derived lemmas. Primitive constructions used in the system are always non-instantiated.

The search procedure starts from the given points and tries to construct all three vertices of the triangle. Primitive constructions are used to construct new objects from the current ones: if some primitive construction can be instantiated so that all objects from its preconditions are already constructed, but its resulting object is not yet constructed, then the object is added to the set of constructed objects. Let us consider the primitive construction saying that for two given points one can construct the line passing through them; if, for instance, the circumcenter $O$ of the triangle $A B C$ and the midpoint $M_{a}$ of the side $B C$ are already constructed, but not the perpendicular bisector $m_{a}$ of the side $B C$, we can use this primitive construction, construct the line $m_{a}$ and add it to the set of constructed objects. After successful application of one primitive construction, the search for the next applicable primitive construction starts from the beginning. If all three vertices of triangle are constructed, the search succesfully finishes. If no primitive construction is applicable and at least one of the vertices is not yet constructed, the problem is declared unsolvable using given knowledge base (however, it does not mean that it is not constructible given ruler and compass). Some additional techniques are employed in order to make the search process more efficient. For instance, only relevant objects are constructed - a midpoint of a segment determined by two known points is constructed only if it appears in a definition or a lemma involving some object, not yet constructed. Therefore, definitions and lemmas guide the construction process. After the construction is automatically generated, it is simplified and all irrelevant steps that are not used for the construction of triangle vertices are eliminated. 


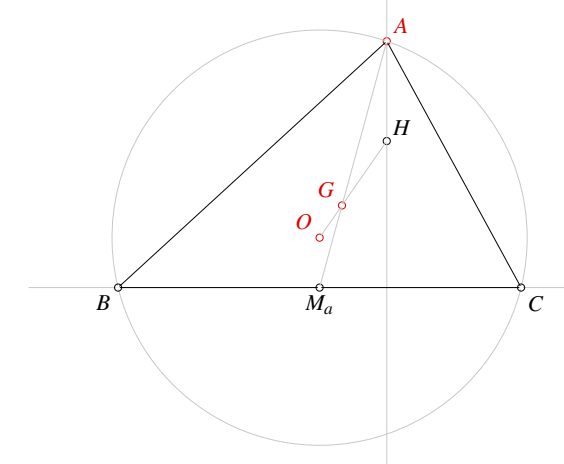

Figure 2: Construction of triangle $A B C$ in Euclidean geometry given the vertex $A$, circumcenter $O$, and centroid $G$.

Example 1 Let's take a look at one construction automatically found by ArgoTriCS. The problem is to construct triangle $A B C$ given vertex $A$, circumcenter $O$ and its centroid $G$. The sequence of construction steps is the following (see Figure 2):

Step 1. Construct the point $M_{a}$ for which holds $\overrightarrow{A M_{a}} / \overrightarrow{A G}=3 / 2$;

Step 2. $\quad$ Construct the point $H$ for which holds $\overrightarrow{O H} / \overrightarrow{O G}=3$;

Step 3. Construct the line $h_{a}$ through the points $A$ and $H$;

Step 4. $\quad$ Construct the circle $\kappa$ centered at point $O$ passing through point A;

Step 5. $\quad$ Construct the line a perpendicular to the line $h_{a}$ passing through point $M_{a}$;

Step 6. Construct the intersection points $B$ and $C$ of the circle $\kappa$ and the line $a$.

The facts used for generating this solution are that centroid $G$ divides the median $A M_{a}$ in the ratio 2:1 and that centroid $G$ is between circumcenter $O$ and orthocenter $H$ and twice as far from the orthocenter than from the circumcenter.

Solving time of different construction problems differ a lot: for most problems it is a couple of milliseconds, while for some (e.g. locus dependent problems) it can last more than an hour. Most of the generated constructions are the same as the ones found in literature.

\subsection{Primitive constructions for Euclidean geometry}

Here we list a few primitive constructions that ArgoTriCS uses for generating constructions in the framework of Euclidean geometry. Note that high-level constructions (such as dropping a perpendicular or constructing a parallel) can be expressed in terms of basic, low-level RC-constructions.

1. Given points $X$ and $Y$ one can construct a line $X Y$;

2. Given two distinct points $X$ and $Y$ it is possible to construct a circle centered at point $X$ which passes through the point $Y$;

3. Given two lines, it is possible to construct their intersection point;

4. Given a point $X$ and a line $p$ one can construct a line $q$ which passes through the point $X$ and which is perpendicular to the line $p$;

5. Given points $X$ and $Y$ one can construct the bisector of the segment $\overline{X Y}$;

6. Given a point $X$ and a line $p$ one can construct a line which passes through the point $X$ and which is parallel to the line $p$; 
7. Given points $X, Z$, and $W$, and a rational number $r$ (given by its numerator and denominator) one can construct a point $Y$ for which holds: $\overrightarrow{X Y} / \overrightarrow{Z W}=r$.

\subsection{Definitions and Lemmas for Euclidean geometry}

Let's take a look at the couple of definitions used by ArgoTriCS.

1. Circumcenter $O$ is the intersection point of the perpendicular bisectors of the segments $B C$ and $A C$;

2. Orthocenter $H$ is the intersection point of the altitudes $h_{a}$ and $h_{b}$;

3. Feet of altitudes $H_{a}, H_{b}$, and $H_{c}$ are intersection points of altitudes with opposite sides of triangle;

4. Circumcircle is the circle centered at circumcenter passing through point $C$.

Here we list a few instantiated and one general lemma used for carrying out constructions in Euclidean geometry and used by ArgoTriCS.

1. Points $C, H_{b}$, and $H_{c}$ belong to the circle centered at point $M_{a}$ passing through point $B$; points $A, H_{a}$, and $H_{c}$ belong to the circle centered at point $M_{b}$ passing through point $C$; points $B, H_{a}$, and $H_{b}$ belong to the circle centered at point $M_{c}$ passing through point $A$;

2. $\overrightarrow{A G} / \overrightarrow{A M_{a}}=2 / 3, \overrightarrow{B G} / \overrightarrow{B M_{b}}=2 / 3, \overrightarrow{C G} / \overrightarrow{C M_{c}}=2 / 3$;

3. Lines $M_{a} M_{b}$ and $A B$ are parallel; lines $M_{a} M_{c}$ and $A C$ are parallel; lines $M_{b} M_{c}$ and $B C$ are parallel;

4. $\angle H A I=\angle I A O, \angle H B I=\angle I B O, \angle H C I=\angle I C O$;

5. Center of an arbitrary circle belongs to the bisector of an arbitrary chord of that circle.

\section{Triangle constructions in non-Euclidean geometries}

In the rest of the text we shall assume the basic knowledge about hyperbolic geometry and its models (e.g., Poincaré disk model). For more details on hyperbolic geometry and its relations to Euclidean geometry we refer the readers to classic literature [4, 12]. Also, for the avid readers, we strongly recommend reading the original works of Lobachevsky and Bolyai.

First, let us emphasize the relation between absolute geometry and the Euclidean or hyperbolic setting. It is well known that the absolute geometry is based on four groups of axioms: incidence, order, congruence, and continuity. By adding the appropriate axiom of parallelism, we get either Euclidean or hyperbolic geometry.

Recall that the question of parallelism is basically the following: In Euclidean geometry there is a unique line parallel to a given line $a$ through a point $A$ not on the line, while in hyperbolic geometry there are infinitely many parallels to $a$ through a point $A$. However, there are exactly two parallel lines that contains the limiting parallel rays (in Poincaré disc model those are h-lines meeting only at the infinity, i.e. on the absolute) and those two lines we call parallel to a line $a$. All the other lines not intersecting line $a$ are called hyperparallel. For example, a line through point $A$ that is parallel/hyperparallel to line $B H_{b}$ is the line $A H_{a}$ from the middle/right picture of Figure 3 , respectively.

Many statements related to the geometry of a triangle can be proved without a notion of parallelism. Let us mention some of the main ones:

1. The sum of internal angles of a triangle is less or equal to $\pi$.

2. The three medians of a triangle intersect in one point (the centroid $G$ ).

3. The three internal angle bisectors of a triangle intersect in one point (the incenter $I$ ).

4. The perpendicular bisectors of triangle sides belong to the same pencil of lines (see [4]). The same holds for the altitudes of a triangle. 
In the Euclidean case, the sum of the internal angles of each triangle is exactly $\pi$, the perpendicular bisectors of triangle sides are concurrent lines that intersect in the circumcenter $O$ and altitudes are also concurrent and intersect in the orthocenter $H$. In the hyperbolic case, the sum of the internal angles of each triangle is always less than $\pi$. Also, unlike the Euclidean case, similar triangles are always congruent. As illustrated in Figure 3 , in the hyperbolic case the altitudes of the triangle may not intersect. The same holds for the perpendicular bisectors. Namely, altitudes/perpendicular bisectors of triangle belong to the same pencil of lines. In the Euclidean case, this pencil is always elliptic, i.e. the lines are concurrent, but in the hyperbolic case, it can also be parabolic (lines are parallel) or hyperbolic (lines are hyperparallel, i.e. they poses a common perpendicular).
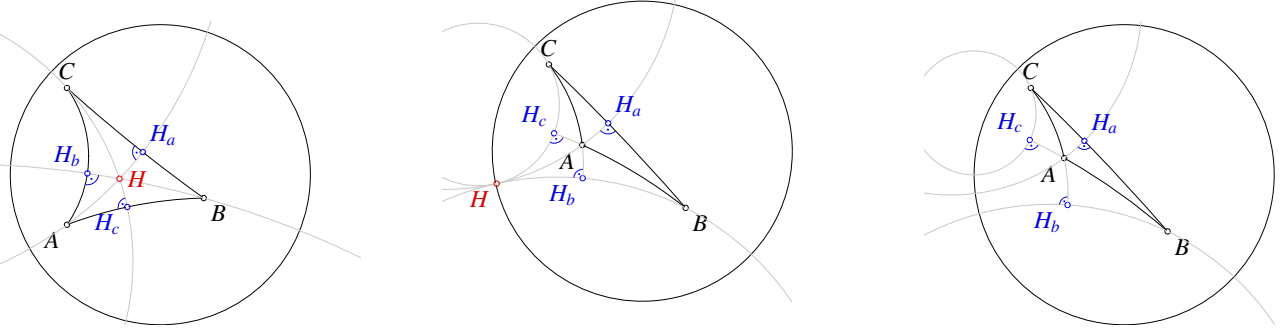

Figure 3: Elliptic, parabolic, and hyperbolic pencil of altitudes.

Therefore, one can expect many similarities, but also many differences between constructions in Euclidean and hyperbolic geometry.

Hyperbolic instruments. Ruler and compass can be used to draw points, straight lines, and circles, and these are the most important loci of points in Euclidean geometry. For example, the set of points on one side of a line, equidistant from that line forms another line (parallel to the original). All vertices of angles of given size subtending a line segment lie on a circular arc. In hyperbolic geometry there are other types of curves that can be of interest. For example, the equidistant curve is not a line, but a hypercycle (Figure 4). Another useful curve type is horocycle (Figure 5) whose normal lines all converge asymptotically in the same direction (in the Euclidean case horocycles are lines, and all normal lines of a horocycle are parallel and thus converge in the same direction).

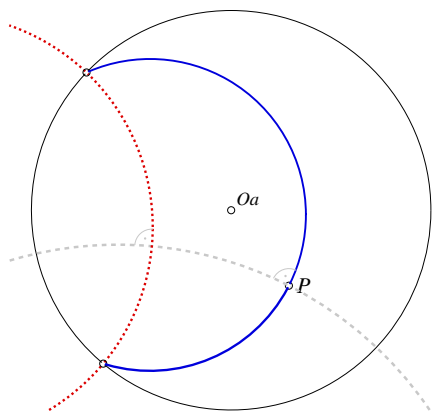

Figure 4: Hypercycle (solid blue) through point $P$ with its axis (dotted red) and normal (dashed)

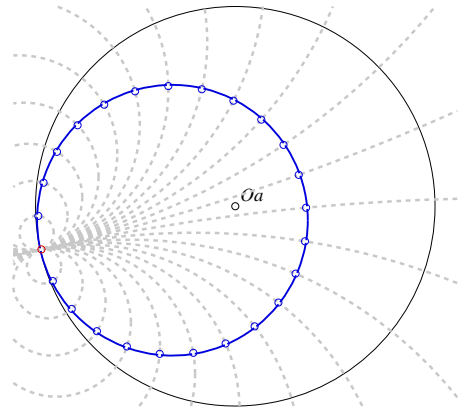

Figure 5: Horocycle (solid blue) and its perpendicular lines (dashed)

Therefore, it is very important to make precise what instruments can be used in a construction and what curves can be constructed. Various hyperbolic instruments have been proposed [2]. The hyperbolic ruler draws a h-line given its two different points, the hyperbolic compass draws a h-circle given its center and a point, the hypercompass draws a hypercycle given its central line and radius, and the horocompass 
draws a horocycle through a given point, given its diameter through the point with its direction. An important (and a bit surprising) result in the theory of hyperbolic constructions shows that in conjunction with a hyperbolic ruler, all three compasses are equivalent (everything that can be constructed by a hyperbolic ruler and one of these three compasses can also be constructed by a hyperbolic ruler and any other one of these three compasses) [13, 4]. Using a combination of a hyperbolic ruler and any of the three compasses one can also draw a h-line through a given point parallel to a given h-ray and a h-line parallel to one and perpendicular to the other given h-line [7, 2].

Additional confusion can come from the fact that in some models of hyperbolic geometry circles represent many different hyperbolic curves. For example, in the Poincaré disc model h-lines are represented by Euclidean circular arcs that are orthogonal to the unit circle (absolute), h-circles are represented by Euclidean circles fully contained within the absolute (however, the h-center does not need to match the Euclidean center), hypercycles are represented by Euclidean circular arcs that are not orthogonal to the unit circle, and horocycles are represented by Euclidean circles touching the absolute from inside. Therefore most diagrams in the Poincaré model can be drawn using Euclidean ruler and compass. However, such constructions are not intrinsic constructions of hyperbolic geometry. In other models, things are quite different. For example, in the Beltrami-Klein model h-lines are Euclidean segments (chords of the absolute), but h-circles are Euclidean ellipses.

Pseudo-elements. At first sight, many fundamental theorems of Euclidean geometry fail to hold in hyperbolic geometry. For example, in Euclidean geometry the orthocenter, the circumcenter and the centroid of a triangle are collinear and lie on a so called Euler line of the triangle. Under standard definitions of medians and altitudes, this does not hold in hyperbolic geometry (moreover, orthocenter and circumcenter may not even exist). However, it turns out that in the Euclidean case many notions can be defined in equivalent ways.

For example, a median is the segment that connect a triangle vertex with the midpoint of its opposite side, but is also a segment that divides the triangle area in two exact halves. In Euclidean geometry those two notions coincide and the same term is used in both cases. However, since in hyperbolic geometry these notions are different, segments that connect triangle vertices with midpoints of opposite triangle sides are usually called median ${ }^{3}$, and segments that divide triangle area in halves are called pseudomedians [1]. Similarly, altitudes are defined using lines perpendicular to the opposite triangle sides. However, they could be also defined by noting that the altitude feet $H_{a}, H_{b}$, and $H_{c}$ are three unique points on triangle sides $B C, A C$, and $A B$ such that the quadrilaterals $A B H_{a} H_{b}, A C H_{a} H_{c}$, and $B C H_{b} H_{c}$ are all cyclic. The second definition gives the notion of pseudoaltitudes (Figure 6).

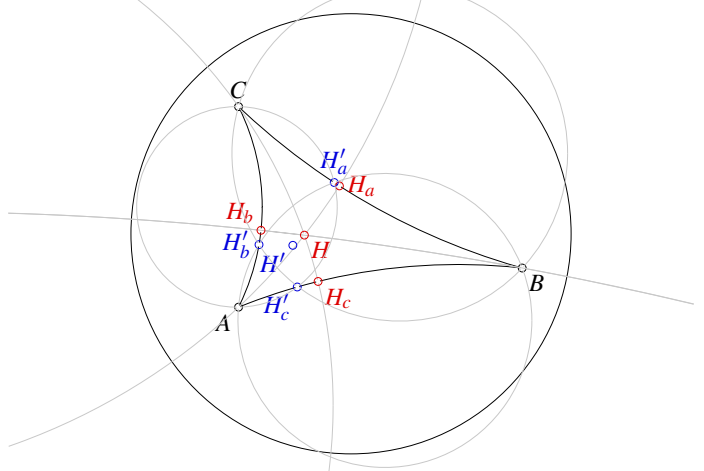

Figure 6: Alternative definitions of altitudes: orthocenter $H$ and pseudoorthocenter $H^{\prime}$

\footnotetext{
${ }^{3}$ To be more precise, these are h-medians as they connect vertices with h-midpoints of opposite sides.
} 
The pseudoorthocenter (intersection of pseudoaltitudes), the circumcenter, and the pseudocentroid (intersection of pseudomedians) are always collinear and lie on a line that deserves to be called the (pseudo) Euler line [1]. This suggests that many Euclidean constructions that use the Euler line could be transferred to hyperbolic geometry, if alternative definitions are used. We advocate that hyperbolic triangle has more significant points than Euclidean. Some significant points that are different in hyperbolic geometry coincide in Euclidean geometry (for example, a hyperbolic triangle has both the orthocenter and pseudoorthocenter, but they always coincide for Euclidean triangles). Therefore, Wernick's corpus should be extended taking into account many different characterizations of points and notions that coincide in Euclidean, but are different in hyperbolic geometry. This way, we may not only consider the pseudo versions of the listed problems, but we may also include the combinations of regular and pseudo-points (e.g. construct a triangle given both the orthocenter and the pseudoorthocenter).

An important challenge is to determine RC-constructibility and construction procedures for such extended set of points. For example, while it is easy to construct the classic centroid of a hyperbolic triangle (as an intersection of h-medians) it is challenging (if possible at all) to construct its pseudocentroid. The same holds for pseudoorthocenter. Since our main focus at this point is to consider problems that can be easily RC-constructed, the pseudo objects will not be subject of the present research.

\section{Automated triangle construction in non-Euclidean geometries}

\subsection{Primitive constructions}

Which primitive constructions can be transferred from Euclidean to hyperbolic geometry? If we take a closer look at the list given in Subsection 2.1, we see that the primitive construction 7 cannot be carried out in the hyperbolic case. The notion of ratio of collinear points must be substituted by the cross-ratio of collinear points. However, in a majority of solutions, we only use two special cases: for the fixed points $X$ and $Y$ construct a point $Z$ that is either the midpoint of the segment $X Y$ or is symmetric to the point $X$ wrt. the point $Y$. Since these constructions can be performed in hyperbolic geometry, they will substitute the more general Euclidean one without much loss:

77. Given points $X$ and $Y$ construct the midpoint $Z$ of the segment $X Y$;

7b. Given points $X$ and $Y$ construct the point $Z$ symmetric to $X$ wrt. point $Y$.

For example, the first construction is used to construct point $M_{a}$ given the points $B$ and $C$, while the second one is necessary when points $B$ and $M_{a}$ are given and we want to construct point $C$.

The rest of the list of primitive constructions given in Section 2.1 can be carried out in the hyperbolic case as well with one exception: the primitive construction 6 does not have a unique solution in the hyperbolic case (there are two lines through a given point parallel to the given line). Additionally, we can construct infinitely many lines containing the given point that have no intersection with the given line, i.e. the construction of the hyperparallel lines is also possible. However, the need to construct an arbitrary line through a point that diverges from a given line rarely arises. Usually, we need to choose a specific line out of this set. But what choice is natural to make? One of the characterizations of hyperparallel lines is that they have a unique common perpendicular. Therefore, we can say that we know how to construct a line $b \ni A$ hyperparallel to $a$ such that the given point $A \notin a$ is the foot of the common perpendicular of $a$ and $b$.

Now, in the hyperbolic case, we have two primitive constructions substituting the primitive construction 6

6a. Construct the line $p$ through point $P$ that is parallel in the given direction to the line passing through points $X$ and $Y$; 
6b. Construct the line $p$ through point $P$ that is hyperparallel to the line passing through $X$ and $Y$, with $P$ being the foot of their common perpendicular.

Further, we can extend the list with two new primitive constructions that are also applicable in the Euclidean case. These were not previously used since they were subsumed by constructions based on stronger statements specific to the Euclidean setting (for illustration, see Example 3). However, in absolute geometry, it is natural to consider the image of some object under the reflection wrt. the given line, since every plane isometry can be represented as a composition of a finite number of reflections. Hence, we add the following:

8. Given line $m$ and point $P$, one can construct a point which is an image of point $P$ under the reflection wrt. line $m$;

9. Given point $M$ and line $p$, one can construct a line which is an image of line $p$ under the reflection wrt. point $M$.

Note that the reflection wrt. the point can be represented as a composition of two reflections wrt. the perpendicular lines intersecting in that point.

\subsection{Definitions and Lemmas}

Definitions of the basic elements are given in terms of absolute geometry, hence they can be transferred from the Euclidean case. However, one should always have in mind that when saying "line" we are thinking of "h-line". The illustration of significant points from Wernick's corpus in the hyperbolic setting is given in Figure 7 .

Although some of the lemmas from the Euclidean case can be transferred to the hyperbolic setting (see Lemma 5), most of them needs to be discarded or adapted.

For example, the lemmas referring to the ratio of segments, especially the ones related to the centroid $G$ of the triangle, do not hold in the hyperbolic case, hence the construction presented in the Example 1 is not possible in the hyperbolic setting. Despite the median concurrence theorem being true, the ratios from Lemma 2 do not hold. Also, Lemma 1 will hold only in the pseudo-case.

On the other hand, in the Euclidean case the midline of the triangle is parallel to the corresponding side of the triangle, while in the hyperbolic case those lines are hyperparallel. Hence, the Lemma 3 has its hyperbolic version:

$3^{h}$ Lines $M_{a} M_{b}$ and $A B$ are hyperparallel and $M_{c}$ is the foot of their common perpendicular; lines $M_{a} M_{c}$ and $A C$ are hyperparallel and $M_{b}$ is the foot of their common perpendicular; lines $M_{b} M_{c}$ and $B C$ are hyperparallel and $M_{a}$ is the foot of their common perpendicular.

Similarly, we know that the line $A I$ is a bisector on an angle $\angle A^{\prime} A O$, where $A^{\prime}$ is the foot of perpendicular from point $A$ to the midline $M_{b} M_{c}$. In the Euclidean case, points $A, A^{\prime}$ and $H$ are collinear, while in the hyperbolic one they are not. Hence, Lemma 4 cannot be used in the present form.

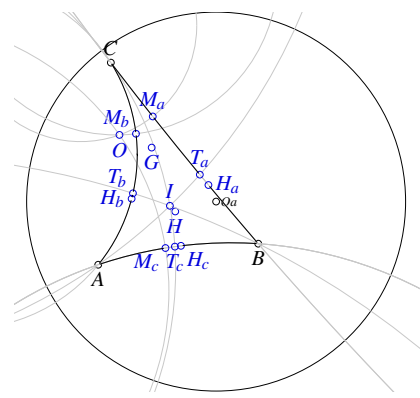

Figure 7: Significant points from Wernick's corpus in hyperbolic geometry. 
We added some more lemmas which relate to notion of reflection with respect to point/line. All of them hold, also, in Euclidean geometry, but we didn't need them there since we found alternative solutions to construction problems.

1. If a vertex $A$ of triangle $A B C$ belongs to the line $p$, then a vertex $B$ belongs to a line which is an image of line $p$ under the reflection wrt. point $M_{c}$;

2. Image of the vertex $B$ under the reflection wrt. internal angle bisector $s_{c}$ belongs to line $A C$;

3. Image of the vertex $B$ under the reflection wrt. internal angle bisector $s_{c}$ belongs to the circle centered at point $T_{c}$ passing through point $B$.

\subsection{Examples}

Here, we give couple of examples illustrated in Poincaré disc model. Note that all constructions can be performed using only classical ruler and compass, since all the basic objects (h-lines, h-circles, etc.) are either Euclidean lines or circles.

All generated solutions of problems from Wernick's corpus in the Euclidean setting can be found online at http://www.matf.bg.ac.rs/ vesnap/animations/compendium_wernick.html, and in the hyperbolic setting at http://www.matf.bg.ac.rs/ vesnap/animations_hyp/compendium_wernick_hyperbolic.html.

Example 2 Let us start with the most basic construction problem that illustrates the difference between Euclidean and hyperbolic geometry: Construct the triangle $A B C$ given three side midpoints $M_{a}, M_{b}$, and $M_{c}$.

In the Euclidean case, we know that $A B\left\|M_{a} M_{b}, B C\right\| M_{b} M_{c}, A C \| M_{a} M_{c}$. Therefore, it is easy to construct lines $a: M_{a} \in a\left\|M_{b} M_{c}, b: M_{b} \in b\right\| M_{a} M_{c}$, and $c: M_{c} \in c \| M_{a} M_{b}$. Now, points $A, B$, and $C$ are intersection points of lines $b$ and $c, a$ and $c, a$ and $b$, respectively (see Construction 0341).

As already discussed in Section 4.2 in hyperbolic geometry, the notion of parallelism can be replaced with notion of hyper-parallelism. Therefore, we will use the construction of hyperparallel lines from Lemma 3 (see Figure 8 and Construction 0341).

Step 1. Construct the line a that is hyperparallel to the line through points $M_{b}$ and $M_{c}$ with point $M_{a}$ being the foot of their common perpendicular;

Step 2. $\quad$ Construct the line $b$ that is hyperparallel to the line through points $M_{a}$ and $M_{c}$ with point $M_{b}$ being the foot of their common perpendicular;

Step 3. Construct the intersection point $C$ of the lines a and $b$;

Step 4. Construct the point $B$ symmetric to $C$ wrt. point $M_{a}$;

Step 5. Construct the point A symmetric to $C$ wrt. point $M_{b}$.

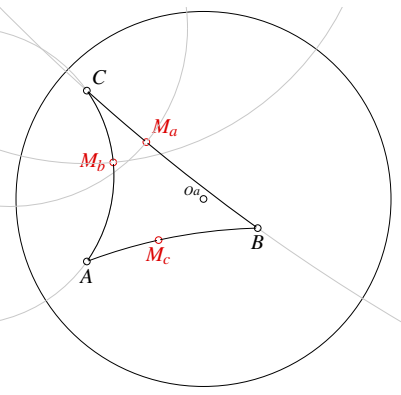

Figure 8: Construction of triangle $A B C$ in hyperbolic geometry given the side midpoints $M_{a}, M_{b}$, and $M_{c}$. 
Example 3 Let us consider a construction of triangle $A B C$ given vertex $A$, midpoint $M_{a}$ of side BC, and foot $H_{b}$ of the altitude from vertex $B$.

This is one of the easiest problems to solve in the Euclidean case, requiring only four construction steps (see Construction 0044). However, the crucial step uses the information that the inscribed angle subtended by a diameter is right. Unfortunately, this does not hold in the hyperbolic case. On the other hand, if we take a closer look at the problem we are trying to solve, we see that we actually need a way to construct a segment with endpoints lying on the chords of the convex angle Opq (not necessarily the right angle) given point $M$ inside that angle as its midpoint. This we know how to construct: we simply need to construct the image $q^{\prime}$ of line $q$ under the reflection wrt. point $M$; the intersection point of $p$ and $q^{\prime}$ will give the first endpoint $P$, while the second one $Q$ will be symmetric to $P$ wrt. point $M$ (see Construction 0044).

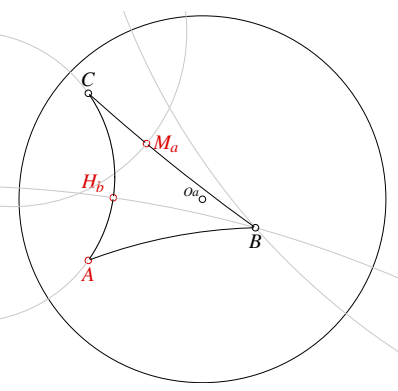

Figure 9: Construction of triangle $A B C$ in hyperbolic geometry given points $A, M_{a}$, and $H_{b}$.

Now, the steps leading to the construction of the triangle ABC in any geometrical setting (i.e. it holds in absolute geometry) are the following:

Step 1. Construct the line $b$ through the points $A$ and $H_{b}$;

Step 2. $\quad$ Construct the line $h_{b}$ perpendicular to the line $b$ through $H_{b}$;

Step 3. Construct the line $s_{M_{a}}(b)$ that is image of the line $b$ under the reflection wrt. point $M_{a}$;

Step 4. Construct the intersection point $B$ of the lines $s_{M_{a}}(b)$ and $h_{b}$;

Step 5. $\quad$ Construct the point $C$ symmetric to $B$ wrt. point $M_{a}$.

\subsection{Results}

We considered only significantly different construction problems from Wernick's list. Immediately, we had to discard all the problems related to the centroid $G$ of the triangle and the ones that had to be solved using Euler's line or Euler's circle. These problems can be solved if we use pseudo-definitions of those objects, but in the traditional setting, we could not find an alternate solution for them. Solutions of all problems from Wernick's corpus we managed to solve (including symmetric ones) in hyperbolic setting are given in Hyperbolic corpus. There are 31 significantly different solvable problems, 1 redundant and 11 locus dependent problems.

\section{Conclusions and further work}

We have described the first steps towards an automated system for triangle RC-constructions in absolute and hyperbolic geometry. The same algorithm that was previously used for Euclidean constructions was 
successfully applied within our system ArgoTriCS. However, underlying mathematical knowledge had to be substantially changed by omitting definitions, lemmas and construction steps specific for Euclidean geometry and by adding specifics of hyperbolic geometry.

The present experiment confirms that RC-constructions in absolute and hyperbolic geometry are harder to make than in Euclidean geometry due to less lemmas that can be proved about lines, circles, and significant points of a triangle (hyperbolic triangle has more significant points, that coincide in the Euclidean case). Also, issues of degeneracy and existence of a solution are more complicated than in the Euclidean case, since significant lines in a triangle may not meet (not all pencils of lines are elliptic ones).

In our further work we shall manually investigate problems that were not solved by our current implementation and to extend the knowledge base (lemmas and primitive steps) so that the problems that can be solved manually could also be solved automatically. We also plan to investigate potential use of our system in mathematical education, since studying Euclidean and hyperbolic geometry and their relationship can help students to acknowledge and adopt formal mathematics.

\section{References}

[1] Arseniy V. Akopyan (2011): On some classical constructions extended to hyperbolic geometry. arXiv 1105.2153 .

[2] MW Al-Dhahir (1962): An instrument in hyperbolic geometry. Proceedings of the American Mathematical Society 13(2), pp. 298-304, doi 10.4153/CJM-1956-043-0.

[3] Bruno Buchberger (2006): An algorithm for finding the basis elements of the residue class ring of a zero dimensional polynomial ideal. Journal of Symbolic Computation 41(3), pp. 475-511, doi $10.1016 /$ j.jsc.2005.09.007.

[4] H. S. M. Coxeter (1957): Non-euclidean geometry. Univ. of Toronto Press, Toronto, doi $10.3138 / 9781442653207$

[5] Xiao-Shan Gao \& Shang-Ching Chou (1998): Solving geometric constraint systems. II. A symbolic approach and decision of RC-constructibility. Computer Aided Design 30(2), pp. 115-122, doi:10.1016/S00104485(97)00055-9.

[6] Sumit Gulwani, Vijay Anand Korthikanti \& Ashish Tiwari (2011): Synthesizing Geometry Constructions. In: Proceedings of the 32nd ACM SIGPLAN Conference on Programming Language Design and Implementation, pp. 50-61, doi:10.1145/1993498.1993505.

[7] F. Handest (1956): Constructions in hyperbolic geometry. Cañad. J. Math. 8, pp. 389-394, doi:10.4153/CJM1956-043-0.

[8] Predrag Janičić (2010): Geometry Constructions Language. Journal of Automated Reasoning 44(1-2), pp. 3-24, doi:10.1007/s10817-009-9135-8

[9] Vesna Marinković \& Predrag Janičić (2012): Towards Understanding Triangle Construction Problems. In: Intelligent Computer Mathematics - CICM 2012, Lecture Notes in Computer Science 7362, pp. 126-141, doi: $10.2307 / 2690164$

[10] Vesna Marinković (2017): ArgoTriCS - Automated Triangle Construction Solver. Journal of Experimental \& Theoretical Artificial Intelligence 29(2), pp. 247-271, doi:10.2307/2690164

[11] Filip Marić, Ivan Petrović, Danijela Petrović \& Predrag Janičić (2012): Formalization and Implementation of Algebraic Methods in Geometry. In: Proceedings First Workshop on CTP Components for Educational Software, Electronic Proceedings in Theoretical Computer Science 79, pp. 63-81, doi:10.4204/EPTCS.79.4.

[12] John Milnor (1982): Hyperbolic geometry: The first 150 years. Bulletin of the American Mathematical Society 6, pp. 9-24, doi:10.2307/1971277. 
[13] D.D. Mordoukhay-Boltovskoy (1927): About geometrical constructions in Lobacevskii space (in Russian). JFM 53.0592.01, pp. 67-82.

[14] Victor Pambuccian (2008): Axiomatizing geometric constructions. Journal of Applied Logic 6(1), pp. 24-46, doi: $10.1016 /$ j.jal.2007.02.001.

[15] Pascal Schreck (1993): Constructions à la règle et au compas. Ph.D. thesis, University of Strasbourg.

[16] Pascal Schreck (2019): On the Mechanization of Straightedge and Compass Constructions. Journal of Systems Science and Complexity 32, pp. 124-149, doi 10.1007/s11786-016-0255-3.

[17] Pascal Schreck, Pascal Mathis, Vesna Marinković \& Predrag Janičić (2016): Wernick's List: A Final Update. Forum Geometricorum 16, pp. 69-80.

[18] William Wernick (1982): Triangle Constructions with Three Located Points. Mathematics Magazine 55(4), pp. 227-230, doi:10.1080/0025570X.1985.11976988.

[19] Wen Tsun Wu (1978): On the Decision Problem and the Mechanization of Theorem-proving in Elementary Geometry. Scientia Sinica 21(2), pp. 159-172, doi:10.1142/9789812791085_0008. 